Revue d'histoire de l'Amérique française

REYUE D.HISTOIRE DE L'AMÉRIQUE FRANÇAISE

\title{
American Problem Studies, under the editorial direction of Oscar Handlin. Holt, Rinehart and Winston, New York, Chicago, San Francisco, Toronto, London, 1962-1967.
}

\section{Georges-Émile Giguère}

Volume 22, numéro 4, mars 1969

URI : https://id.erudit.org/iderudit/302841ar

DOI : https://doi.org/10.7202/302841ar

Aller au sommaire du numéro

Éditeur(s)

Institut d'histoire de l'Amérique française

ISSN

0035-2357 (imprimé)

1492-1383 (numérique)

Découvrir la revue

Citer ce compte rendu

Giguère, G.-É. (1969). Compte rendu de [American Problem Studies, under the editorial direction of Oscar Handlin. Holt, Rinehart and Winston, New York, Chicago, San Francisco, Toronto, London, 1962-1967.] Revue d'histoire de l'Amérique française, 22(4), 652-654. https://doi.org/10.7202/302841ar d'utilisation que vous pouvez consulter en ligne.

https://apropos.erudit.org/fr/usagers/politique-dutilisation/ 
American Problem Studies, under the editorial direction of Oscar Handlin. Holt, Rinehart and Winston, New York, Chicago, San Francisco, Toronto, London, 1962-1967.

Le procédé de présentation de l'histoire sous forme de problèmes est peut-être plus ou moins récent, mais à cause de son excellence et de la faveur dont il jouit, il se pratique de plus en plus. C'est probablement la meilleure façon d'enseigner et d'étudier l'histoire. Manière plus dynamique qui procède par questions et réponses, au lieu de fournir d'abord la solution, et de laisser au professeur la tâche de poser les questions. C'est ainsi mettre fin à l'histoire statique qui a causé tant de dommages. 
L'introduction de cet ouvrage n'est rien d'autre qu'un excellent résumé du problème et des différentes tendances remarquées chez les auteurs qui ont traité le même sujet. Prenons l'exemple de la Révolution américaine. Le premier texte est de Georges Bancroft qui en 1834 a présenté cette révolution comme une lutte de la liberté contre la tyrannie. Trois écoles ont par la suite interprété les causes de la Révolution avant que les révisionnistes fassent la critioue de ces interprétations. Quant aux conséquences, les écoles se sont rangées soit du côté révolutionnaire soit du côté conservateur. Ce n'est là qu'une illustration, car en parcourant la collection, on constate que les auteurs se répartissent en impérialistes et anti-impéralistes, en économistes et sociologues, sans compter la critique qui réévalue les écoles antérieures. Les témoignages qui s'établissent entre 12 et 15 par question ou par sujet ont pris place à des époques différentes et contiennent diverses idéologies sous-jacentes, démocratiques ou antidémocratiques, sans compter les divergences religieuses.

Le plan de l'ouvrage varie selon les sujets. Il est composé d'un ensemble d'extraits empruntés aux divers historiens qui ont traité ce problème à différentes époques. Le grand avantage de ce procédé, et c'est peut-être la seule conclusion qu'on en peut tirer, est le pluralisme des opinions et des interprétations. L'endoctrinement selon une seule optique est ainsi évité et avec lui le dirigisme idéologique. Il en découle un grand respect et une grande confiance envers l'étudiant. Il élimine l'impression statique et autoritaire qu'on aurait pu avoir de l'histoire en montrant son dynamisme et sa relativité. C'est évidemment mettre fin à l'escalade vers l'autoritarisme des interprétations, c'est également enrayer le mythe des prophètes et des pontifes qui fulminent l'excommunication, c'est dissoudre les chapelles et détruire à l'avance toute forme de monolithisme.

Cette méthode a également l'avantage de dégager un problème en l'isolant des complications d'une conjoncture chronologique souvent trop lourde. Bien loin de dispenser de l'étude chronologique ou événementielle de l'histoire, elle y pousse tout en orientant plus nettement vers les philosophies sous-jacentes qui orientent et inspirent souvent les interprétations et qui différencient entre eux les historiens, les écoles et les tendances. En rapprochant les opinions sans toutefois les opposer, elle révèle la richesse d'une histoire et la multiplicité de ceux qui, à une époque ou à l'autre, l'ont scrutée dans une optique différente. Elle conduit indispensablement chacun à un retour vers les faits, les événements, en l'obligeant à rétablir par lui-même une stricte 
chronologie toujours nécessaire à la formation d'un jugement plus personnel, et partant plus attrayant, plus satisfaisant parce que plus stimulant.

Le plan de la thèse de la "frontière" en fournira une illustration en étalant l'évolution du problème depuis ses débuts: the beginning of the controversy - the frontier as a safety valve drawing excess wage earners from the east - democracy as a product of the frontier - influence of the frontier on american character.

Chacun des ouvrages de cette collection compte 122 pages et se termine par un guide de lectures qui est en somme une bibliographie commentée, selon une pratique fréquente dans le monde américain ou anglais. Cette partie a pour but de suggérer des lectures destinées à compléter l'information du lecteur ou de l'étudiant.

L'ensemble de chaque fascicule a été confié à un spécialiste dont la science très sûre se révèle aux indications qu'il fournit. Il convient en terminant d'énumérer la liste des ouvrages parus dans cette intéressante série:

Puritanism in Seventeenth-Century Massachusetts - David D. Hall

Politics and Society in Colonial America - Michael G. Kammen The American Revolution - George Athan Billias

The Federalists vs the Jeffersonian Republicans - Paul Goodman

The Causes of the War of 1812 - Bradford Perkins

The Monroe Doctrine - Armin Rappaport

Jacksonian Democracy - James L. Bugg, jr

The Abolitionists - Richard O. Curry

The Mexican War-Ramon Eduardo Ruiz

The Frontier Thesis - Ray Allen Billington

American Expansion in the Late Nineteenth Century - J. Rogers Hollingsworth

The Progressive Era - Arthur Mann

America's Entry into World War I - Herbert J. Bass

Wilson and the League of Nations - Ralph A. Stone

The New Deal - Morton Keller

The Atomic Bomb - Paul R. Baker.

Directeur

Institut Albert-Tessier

Trois-Rivières
GeORGES-Émile GigUÈre, s.j. 